\title{
Structural Health Monitoring of Marine Structures by Using Inverse Finite Element Method
}

\author{
A. KEFAL \& E. OTERKUS \\ Department of Naval Architecture, Ocean and Marine Engineering, University of Strathclyde, Glasgow, \\ United Kingdom
}

\begin{abstract}
A new state-of-the art methodology named as inverse Finite Element Method (iFEM) is adopted to solve the inverse problem of real-time reconstruction of full-field structural displacements, strains, and stresses. iFEM has shown to be precise, robust, and fast enough to reconstruct the three dimensional displacement field of structures in real-time by utilizing surface strain measurements obtained from strain sensors embedded on the structure. The numerical implementation of the iFEM methodology is done by considering four-node inverse quadrilateral shell element. Two demonstration cases are presented including a quadrilateral plate subjected to bending force and a stiffened plate under bending loading. Finally, the effect of sensor locations, number of sensors and the discretization of the geometry are examined on solution accuracy.
\end{abstract}

\section{INTRODUCTION}

Well-maintained structures are more durable. Increase in durability decreases the direct economic losses (repair, maintenance, reconstruction) and also helps to avoid losses for users that may suffer due to a structural malfunction. Furthermore, new materials, new construction technologies and new structural systems are increasingly being used in marine industry; therefore it is necessary to increase knowledge about their on-site structural condition and integrity. Structural health monitoring (SHM) certainly provides satisfactory answers to these requests.

Reconstruction of the full-field structural deformations, strains and stresses is a key component of SHM by utilizing the strain data obtained from a network of on-board strain sensors located at various sites of a structure (Tessler \& Spangler 2005). A regularization term which ensures a certain degree of smoothness to solve this inverse problem was introduced by Tikhonov \& Arsenin (1977) and most of the today's inverse algorithms uses Tikhonov's regularization (refer to Liu \& Lin 1996, Maniatty \& Zabaras 1994, Maniatty et al. 1989, Schnur \& Zabaras 1990 and references therein). However, most of these inverse methods are not generally appropriate for use in on-board SHM procedures because many of these didn't take into consideration the complexity of boundary conditions and structural topology. Moreover, they mostly require adequately precise loading and/or material information, although it shouldn't be the case for a powerful SHM algorithm.
A new state-of-the art methodology named as inverse Finite Element Method (iFEM), which certainly satisfies the necessities of SHM procedure, was developed by Tessler \& Spangler (2003, 2005). iFEM algorithm reconstructs the structural deformations from experimentally measured strains based on the minimization of a weighted-least-square functional. Unlike other inverse methods, iFEM methodology possesses a general applicability to complex structures subjected to complicated boundary conditions in real-time (Tessler \& Spangler 2005). iFEM framework is precise, powerful and sufficiently fast for real-time applications of any type of static and dynamic loadings, as well as a wide range of elastic materials since only strain-displacement relationship is used in the formulation (Gherlone et al. 2012, 2014).

The domain of the structural model can be discretized by using beam, frame, or plate and shell inverse finite elements in order to perform SHM based on iFEM algorithm. In order to monitor truss, beam, and frame structures in real-time, Cerracchio et al. (2010) and Gherlone et al. $(2011,2012,2014)$ developed a computationally efficient inverse-frame finite element based on kinematic assumptions of Timoshenko shear-deformation theory. Their numerical and experimental examination of the inverseframe finite element indicated the superiority of iFEM approach for shape-sensing of threedimensional frame structures that are subjected to static or/and damped harmonic excitations. Tessler and Spangler (2004) used first-order shear deformation theory to develop a three-node inverse shell 
element (iMIN3) for analyzing arbitrary plate and shell structures. The precision of iMIN3 element was demonstrated by using experimentally measured strain data by Quach et al. (2005) and Vazquez et al. (2005). Moreover, Tessler et al. (2012) recently improved iMIN3 element formulation to reconstruct deformed shape of plate and shell structures undergoing large displacements. Regarding the application of iFEM analysis on engineering structures, it has been limited to the SHM of aerospace vehicles (Tessler et al. 2011, Gherlone et al. 2013).

The main and novel aim of this study is to demonstrate the applicability of iFEM to SHM of marine structures for the first time in the literature. The presented iFEM formulation is based on the minimization of a weighted-least-square functional that uses Mindlin's first-order plate theory. The numerical implementation of the iFEM methodology is done by developing a four-node inverse shell element (iQS4) including hierarchical drilling rotation degree of freedoms. Various validation and demonstration cases are presented including a quadrilateral plate subjected to bending force and the fundamental problem of a stiffened plate under bending loading which represents the portion of the side of a typical longitudinally and transversely framed tanker. Experimentally measured strains are represented by strain results obtained from a high-fidelity solution using an in-house finite element code. Several types of discretization strategies are examined and comparisons of the reconstructed iFEM and direct FEM displacement solutions are provided. By exploiting the weighting constants in the least-square functional of iFEM, it is confirmed that a relatively accurate deformed structural shape can be reconstructed in the absence of in-situ strain data. Finally, the effect of sensor locations, number of sensors and the discretization of the geometry on solution accuracy is observed.

\section{INVERSE SHELL FINITE ELEMENT FORMULATION}

\subsection{Inverse Quadrilateral Shell Element}

A four-node inverse quadrilateral shell element, labeled as iQS4, having six displacement degrees-offreedom per node as shown in Figure 1 is developed in order to represent the formulation of inverse finite element method. The first step is to define convenient coordinate systems to guarantee the geometric uniqueness of the assembled inverse finite element structure. A local coordinate system xyz is oriented with the reference to the element as shown in Figure 1 to formulate the element stiffness properties.
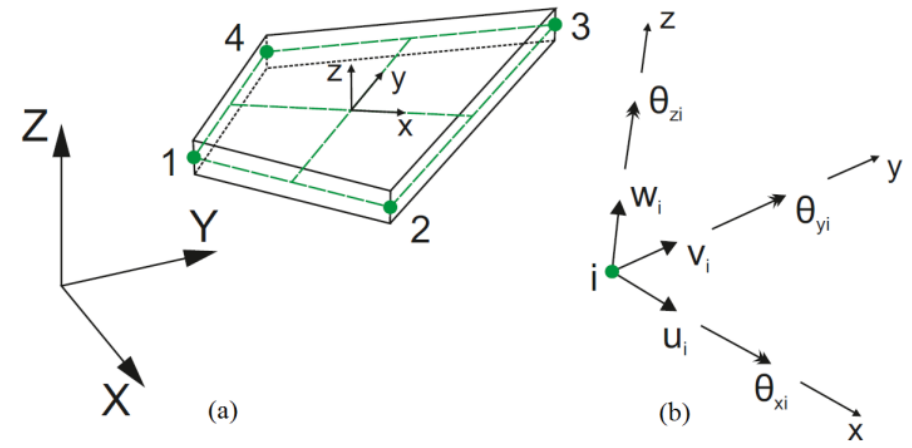

Figure 1. (a) Quadrilateral inverse shell element, showing global and local coordinate systems. (b) Nodal degrees of freedom in the local coordinate system xyz.

By using global XYZ coordinates of the element nodes, transformation matrix of nodal degrees-offreedom of an element from the local to global coordinate system can be established for assembling the elements. As we assume a flat surface for the element, the transformation procedure is straightforward and the details of how to generate the transformation matrix can be found in Bathe (2006).

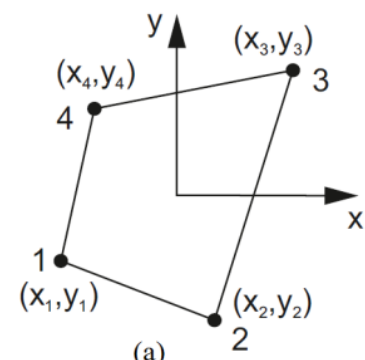

(a)

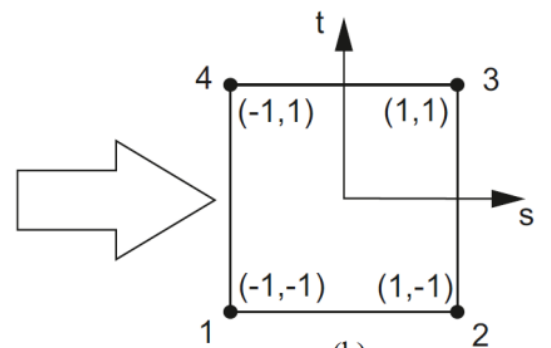

(b)
Figure 2. (a) Quadrilateral inverse shell element projected onto its local xy base plane. (b) Isoparametric coordinates of parent element.

According to the local xy base plane projected view of the iQS4 element and in terms of usual isoparametric coordinates $\mathrm{s}$ and $\mathrm{t}$ as shown in Figure 2, the mapping function of the element can be expressed as following:

$$
\begin{aligned}
& x(s, t)=x=\sum_{i=1}^{4} N_{i} x_{i} \\
& y(s, t)=y=\sum_{i=1}^{4} N_{i} y_{i}
\end{aligned}
$$

where $N_{i}$ are the standard bilinear shape functions and $x_{i}, y_{i}$ are corner coordinates.

The degrees-of-freedoms of $\mathrm{x}$ and $\mathrm{y}$ translations $u_{i}$ and $v_{i}$ together with drilling rotation $\theta_{z i}$, positive counter clockwise, at each corner can be used to express the resulting membrane displacement field $u$ and $v$ given as:

$$
u(x, y)=u=\sum_{i=1}^{4} N_{i} u_{i}+\sum_{i=1}^{4} L_{i} \theta_{z i}
$$


$v(x, y)=v=\sum_{i=1}^{4} N_{i} v_{i}+\sum_{i=1}^{4} M_{i} \theta_{z i}$

where $L_{i}$ and $M_{i}$ are the shape functions consistent with ones proposed by Cook (1994) that defines hierarchical drilling rotation degree of freedom.

Equation $3 \mathrm{a}, 3 \mathrm{~b}$, and $3 \mathrm{c}$ express that the bending displacement field of the element $w, \theta_{x}$, and $\theta_{y}$ are defined by the degrees-of-freedoms of $\mathrm{z}$ translation $w_{i}$ and positive counter clockwise rotations around $\mathrm{x}$ and y axis, $\theta_{x i}$ and $\theta_{y i}$. Isoparametric shape functions $L_{i}$ and $M_{i}$ used to formulate drilling rotation can be utilized to describe the flexural capability of the iQS4 element. Hence, mathematical foundation of bending action of iQS4 element becomes identical to the MIN4 (Mindlin-type, four-nodes) provided by Tessler \& Hughes (1983).

$$
\begin{aligned}
& w(x, y)=w=\sum_{i=1}^{4} N_{i} w_{i}-\sum_{i=1}^{4} L_{i} \theta_{x i}-\sum_{i=1}^{4} M_{i} \theta_{y i} \\
& \theta_{x}(x, y)=\theta_{x}=\sum_{i=1}^{4} N_{i} \theta_{x i} \\
& \theta_{y}(x, y)=\theta_{y}=\sum_{i=1}^{4} N_{i} \theta_{y i}
\end{aligned}
$$

The kinematic relations of the element are prescribed according to the assumptions taken in first order, shear-deformation theory. Using the Equation 1 for membrane action and the Equation 2 for bending action, the three components of the displacement vector of any material point within the element can be described as:

$$
\begin{aligned}
& u_{x}(x, y, z)=u_{x}=u+z \theta_{y} \\
& u_{y}(x, y, z)=u_{y}=v-z \theta_{x} \\
& u_{z}(x, y, z)=u_{z}=w
\end{aligned}
$$

where $u_{x}$ and $u_{y}$ are the average positive in-plane displacements and $u_{z}$ is the displacement across the shell thickness. For the sake of implementation purposes, nodal displacement vector of the element can be stated in compact form by Equation 5 .

$$
\begin{aligned}
& \mathbf{u}^{e}=\left[\begin{array}{llll}
\mathbf{u}_{1}^{e} & \mathbf{u}_{2}^{e} & \mathbf{u}_{3}^{e} & \mathbf{u}_{4}^{e}
\end{array}\right]^{T} \\
& \mathbf{u}_{i}^{e}=\left[\begin{array}{llllll}
u_{i} & v_{i} & w_{i} & \theta_{x i} & \theta_{y i} & \theta_{z i}
\end{array}\right]^{T}(i=1,2,3,4)
\end{aligned}
$$

After taking the relevant derivatives of the three components of the displacement vector given in Equation 4 and utilizing the nodal displacement vector of element $\mathbf{u}^{e}$, the strain-displacement relations of linear elasticity theory can be written in compact vector forms as given in Equation 6. It is important to mention that $\varepsilon_{z z}$ has no role in the internal work due to the plane stress assumption $\sigma_{z z}=0$.

$$
\begin{aligned}
& \left\{\begin{array}{l}
\varepsilon_{x x} \\
\varepsilon_{y y} \\
\gamma_{x y}
\end{array}\right\} \equiv \mathbf{e}\left(\mathbf{u}^{e}\right)+z \mathbf{k}\left(\mathbf{u}^{e}\right)=\mathbf{B}^{m} \mathbf{u}^{e}+z \mathbf{B}^{k} \mathbf{u}^{e} \\
& \left\{\begin{array}{l}
\gamma_{x z} \\
\gamma_{y z}
\end{array}\right\} \equiv \mathbf{g}\left(\mathbf{u}^{e}\right)=\mathbf{B}^{s} \mathbf{u}^{e}
\end{aligned}
$$

In equation 6 , the membrane strains associated with the stretching of the middle surface are $\mathbf{e}\left(\mathbf{u}^{e}\right)$, therefore $\mathbf{B}^{m}$ matrix stands for the derivatives of the shape functions associated with the membrane behavior. Accordingly, the bending curvatures are $\mathbf{k}\left(\mathbf{u}^{e}\right)$, and the transverse shear strains are $\mathbf{g}\left(\mathbf{u}^{e}\right)$ so that $\mathbf{B}^{k}$ and $\mathbf{B}^{s}$ matrices are the corresponding derivatives of shape functions used to define bending behavior of the element.

\subsection{The reference plane strains and curvatures computed from in-situ strain sensors}

Discrete in-situ strain measures obtained from the embedded sensors are crucial according to the iFEM formulation. Conventional strain rosettes or embedded optical-fiber networks such as Fibre Bragg Grating (FBG) sensors are promising technology to collect large amount of on-board strain data. In order to compute the reference plane strains and curvatures, the necessary orientation of the in-situ strain rosettes on iQS4 elements' surface is illustrated in Figure 3.

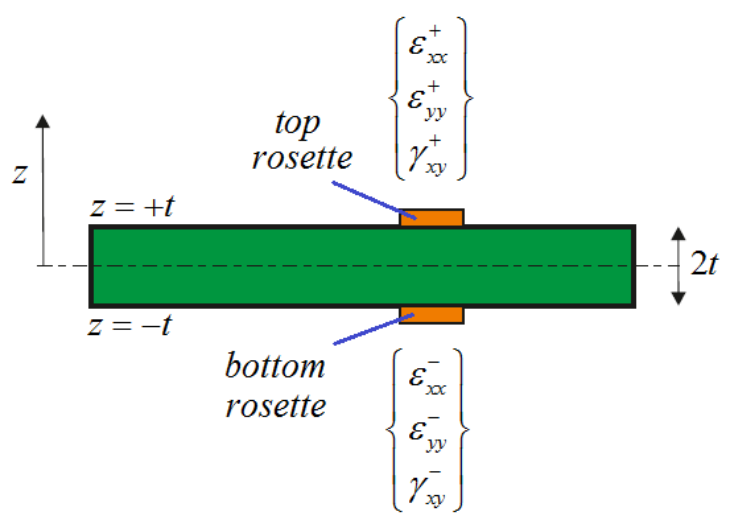

Figure 3. Discrete surface strains measured at location $\mathbf{x}_{i}$ from strain rosettes instrumented on top and bottom of iQS4 elements.

According to Tessler \& Spangler (2005), at $n$ discrete locations $\left(\mathbf{x}_{i}=\mathrm{x}_{i}, \mathrm{y}_{i}, \pm \mathrm{t}\right)(i=1, \ldots, n)$ where the surface strains are measured, the reference plane strains $\mathbf{e}_{i}{ }^{\varepsilon}$, and curvatures $\mathbf{k}_{i}{ }^{\varepsilon}$ that corresponds to the membrane strains $\mathbf{e}\left(\mathbf{u}^{e}\right)$ and bending curvatures $\mathbf{k}\left(\mathbf{u}^{e}\right)$ given in Equation 6 can respectively be determined from the measured surface strains as follows: 


$$
\begin{gathered}
\mathbf{e}_{i}^{\varepsilon}=\frac{1}{2}\left\{\begin{array}{l}
\varepsilon_{x x}^{+}+\varepsilon_{x x}^{-} \\
\varepsilon_{y y}^{+}+\varepsilon_{y y}^{-} \\
\gamma_{x y}^{+}+\gamma_{x y}^{-}
\end{array}\right\}_{i}(i=1, n) \\
\mathbf{k}_{i}^{\varepsilon}=\frac{1}{2 t}\left\{\begin{array}{l}
\varepsilon_{x x}^{+}-\varepsilon_{x x}^{-} \\
\varepsilon_{y y}^{+}-\varepsilon_{y y}^{-} \\
\gamma_{x y}^{+}-\gamma_{x y}^{-}
\end{array}\right\}_{i}(i=1, n)
\end{gathered}
$$

where the measured surface strains are denoted as $\left(\varepsilon_{x x}{ }^{+}, \varepsilon_{y y}{ }^{+}, \varepsilon_{x y}{ }^{+}\right)_{i}$ and $\left(\varepsilon_{x x}{ }^{-}, \varepsilon_{y y}{ }^{-}, \varepsilon_{x y}{ }^{-}\right)_{i}$, superscripts '+' and '-' represent the top and bottom surface locations, and the following notation is used $(\bullet)_{\mathrm{i}}=(\bullet)_{\mathbf{x}=\mathbf{x} \text {. }}$.

Although, the experimentally measured surface strains can be used to compute in-situ membrane strains $\mathbf{e}_{i}{ }^{\varepsilon}$ and bending curvatures $\mathbf{k}_{i}{ }^{\varepsilon}$, they cannot be directly used to calculate in-situ transverse shear strains $\mathbf{g}_{i}^{\varepsilon}$. A smoothing procedure, developed by Tessler et. al $(1998,1999)$, called the Smoothing Element Analysis, enables the first-order derivatives of $\mathbf{k}_{i}^{\varepsilon}$ to be used in computing the transverse shear strains $\mathbf{g}_{i} i$. However, in the deformation of thin shells, contributions of $\mathbf{g}_{i}{ }^{\varepsilon}$ are much smaller compared to the bending curvatures $\mathbf{k}_{i}{ }_{i}$. Since the marine structures such as ship and offshore structures can be modelled by using thin shells, the $\mathbf{g}_{i}{ }^{\varepsilon}$ contributions can be safely omitted in the following formulations.

\subsection{Weighted least-squares functional}

Accounting for the membrane, bending and transverse shear deformations of the individual element, the inverse finite element method reconstructs the deformed shape by minimizing an element functional, namely a weighted least-squares functional $\Phi_{e}\left(\mathbf{u}^{e}\right)$ given in Equation 8a, with respect to the unknown displacement degrees-of-freedom (Tessler \& Spangler 2005). The squared norms expressed in Equation $8 \mathrm{a}$ can be written in the form of the normalized Euclidean norms as given in Equations from $8 \mathrm{~b}$ to $8 \mathrm{~d}$ where $A^{e}$ represents the area of the element.

$$
\begin{aligned}
& \Phi_{e}\left(\mathbf{u}^{e}\right)=w_{e}\left\|\mathbf{e}\left(\mathbf{u}^{e}\right)-\mathbf{e}^{\varepsilon}\right\|^{2}+w_{k}\left\|\mathbf{k}\left(\mathbf{u}^{e}\right)-\mathbf{k}^{\varepsilon}\right\|^{2} \\
& +w_{g}\left\|\mathbf{g}\left(\mathbf{u}^{e}\right)-\mathbf{g}^{\varepsilon}\right\|^{2} \\
& \left\|\mathbf{e}\left(\mathbf{u}^{e}\right)-\mathbf{e}^{\varepsilon}\right\|^{2}=\frac{1}{n} \iint_{A^{e}} \sum_{i=1}^{n}\left(\mathbf{e}\left(\mathbf{u}^{e}\right)_{i}-\mathbf{e}_{i}^{\varepsilon}\right)^{2} d x d y \\
& \left\|\mathbf{k}\left(\mathbf{u}^{e}\right)-\mathbf{k}^{\varepsilon}\right\|^{2}=\frac{(2 t)^{2}}{n} \iint_{A^{e}} \sum_{i=1}^{n}\left(\mathbf{k}\left(\mathbf{u}^{e}\right)_{i}-\mathbf{k}_{i}^{\varepsilon}\right)^{2} d x d y \\
& \left\|\mathbf{g}\left(\mathbf{u}^{e}\right)-\mathbf{g}^{\varepsilon}\right\|^{2}=\frac{1}{n} \iint_{A^{e}} \sum_{i=1}^{n}\left(\mathbf{g}\left(\mathbf{u}^{e}\right)_{i}-\mathbf{g}_{i}^{\varepsilon}\right)^{2} d x d y
\end{aligned}
$$

The weighting constants $w_{e}, w_{k}, w_{g}$ in Equation 8a are positive valued and stand for individual section strains. They control the complete coherence between theoretical strain components and their experimentally measured values. The weighting constants are $w_{e}=w_{k}=w_{g}=1$ for the squared norms given in Equations from $8 \mathrm{~b}$ to $8 \mathrm{~d}$ since in-situ strains $\mathbf{e}_{i}^{\varepsilon}, \mathbf{k}_{i}^{\varepsilon}$, and $\mathbf{g}_{i}{ }^{\varepsilon}$ are assumed to be determined. On the other hand, for any case of missing in-situ strain component, the corresponding weighting constants can be selected as a small positive constant $\alpha=10^{-4}$. Equation 9a restates the squared norm presented in Equation $8 \mathrm{~b}$ for an element that has undetermined in-situ strain component of $\mathbf{e}_{i}^{\varepsilon}$. Accordingly, Equation 8c can be rewritten as Equation $9 \mathrm{~b}$ for an element that has missing the in-situ strain component of bending curvatures $\mathbf{k}_{i}{ }_{i}$. In addition, Equation $8 \mathrm{~d}$ can be updated by Equation 9c for the lack of the transverse shear strains $\mathbf{g}_{i}{ }^{\varepsilon}$.

$$
\begin{aligned}
& \left\|\mathbf{e}\left(\mathbf{u}^{e}\right)\right\|^{2}=\iint_{A^{e}} \mathbf{e}\left(\mathbf{u}^{e}\right)^{2} d x d y \\
& \left\|\mathbf{k}\left(\mathbf{u}^{e}\right)\right\|^{2}=(2 t)^{2} \iint_{A^{e}} \mathbf{k}\left(\mathbf{u}^{e}\right)^{2} d x d y \\
& \left\|\mathbf{g}\left(\mathbf{u}^{e}\right)\right\|^{2}=\iint_{A^{e}} \mathbf{g}\left(\mathbf{u}^{e}\right)^{2} d x d y
\end{aligned}
$$

By virtue of these assumptions, all strain compatibility relations are explicitly satisfied so that Equation $8 \mathrm{a}$ can be minimized with respect to nodal displacement vector as shown in Equation 10. After the minimization, the resultant equation is the element matrix equation $\mathbf{k}^{e} \mathbf{u}^{e}=\mathbf{f}^{e}$ where $\mathbf{k}^{e}$ is element stiffness matrix, $\mathbf{f}^{e}$ is element right-hand-side vector that is a function of the measured strain values, and $\mathbf{u}^{e}$ is the nodal displacement vector of element.

$$
\frac{\partial \Phi_{e}\left(\mathbf{u}^{e}\right)}{\partial \mathbf{u}^{e}}=\mathbf{k}^{e} \mathbf{u}^{e}-\mathbf{f}^{e}=0
$$

Once the element (local) matrix equations are established, the element contributions to the global linear equation system of the discretized structure can be performed as follows:

$$
\begin{aligned}
& \mathbf{K}=\sum_{e=1}^{n e l}\left(\mathbf{T}^{e}\right)^{T} \mathbf{k}^{e} \mathbf{T}^{e} \\
& \mathbf{F}=\sum_{e=1}^{n e l}\left(\mathbf{T}^{e}\right)^{T} \mathbf{f}^{e} \\
& \mathbf{U}=\sum_{e=1}^{n e l}\left(\mathbf{T}^{e}\right)^{T} \mathbf{u}^{e} \\
& \mathbf{K U}=\mathbf{F}
\end{aligned}
$$


where $\mathbf{T}^{e}$ is the transformation matrix of nodal degrees-of-freedom of an element from the local to global coordinate system, $\mathrm{K}$ is global stiffness matrix (symmetric and positive definite matrix), $\mathbf{U}$ is global nodal displacement vector, $\mathbf{F}$ is the global right-hand-side vector (function of the measured strain values), and the script nel stands for total number of inverse elements.

The global stiffness matrix $\mathbf{K}$ includes the rigid body motion mode of the discretized structure; therefore it is a singular matrix. By prescribing problemspecific displacement boundary conditions, the resulting system of equations can be reduced from Equation 11d to the Equation 12a where $\mathbf{K}_{R}$ is a positive definite matrix (always non-singular), and thus it is invertible. Solving the Equation 12a in order to obtain the global displacement degrees-of-freedoms of all nodes $\mathbf{U}_{R}$, is very fast as represented in Equation $12 \mathrm{~b}$ because the matrix $\mathbf{K}_{R}$ needs to be reversed only once because it remains unchanged for a given distribution of strain sensors and is independent of the measured strain values.

$$
\begin{gathered}
\mathbf{K}_{R} \mathbf{U}_{R}=\mathbf{F}_{R} \\
\mathbf{U}_{R}=\mathbf{K}_{R}^{-1} \mathbf{F}_{R}
\end{gathered}
$$

However, the right-hand-side vector $\mathbf{F}_{R}$ is dependent on the discrete surface strain data obtained from insitu strain sensors, and hence it needs to be updated during any deformation cycle. Finally, the matrixvector multiplication $\mathbf{K}_{R}{ }^{-1} \mathbf{F}_{R}$ gives rise to the unknown degrees-of-freedoms vector $\mathbf{U}_{R}$, which provides the deformed structural shape at any real-time.

\section{NUMERICAL RESULTS}

\subsection{Quadrilateral plate}

A quadrilateral plate, whose corner coordinates (in meters) are shown in the Figure 4, is considered to be analyzed. The plate has a uniform thickness of 15 $\mathrm{mm}$ and it is made of steel having the elastic modulus of $210 \mathrm{GPa}$ and Poissons ratio of 0.3. Left edge of the plate is clamped and the upper right corner is subjected to a static transverse concentrated loading in negative $\mathrm{z}$ direction as depicted in Figure 4.

In the beginning, a linear static direct finite element analysis of the plate is performed based on a high-fidelity mesh consisting of 625 quadrilateral shell elements and possessing 10206 degrees-offreedoms by using an in-house finite element code. The resulting direct FEM deflection and rotation are used as a source for the simulated sensor-strain. In other words, the 'experimental' strain measurements used in the following iFEM case analysis are obtained by means of the direct FEM solution.

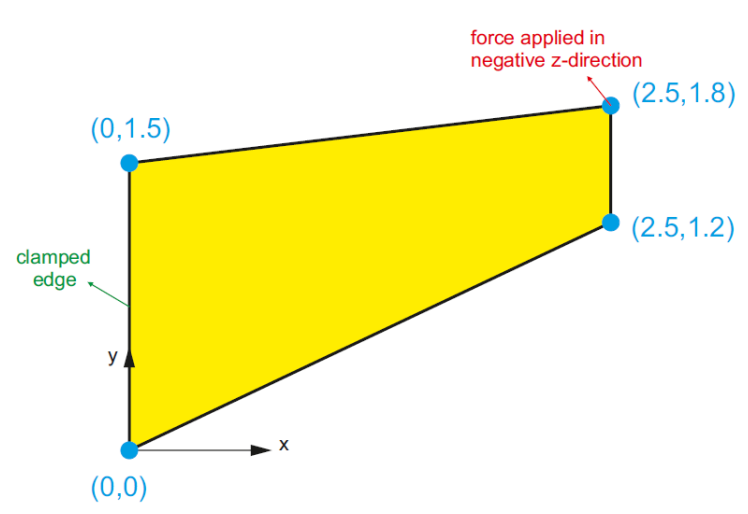

Figure 4. Quadrilateral plate and its boundary condition.

Two SHM case studies of the plate are performed based on iFEM methodology by using different number of strain rosettes and their altered orientation. The strain rosettes can only be placed on one of the bounding surfaces (top or bottom surface) because the material properties of the plate are symmetric with respect to the mid-plane and the resulting deformations are due to bending only so that the strain distribution should be anti-symmetric with respect to the mid-plane.

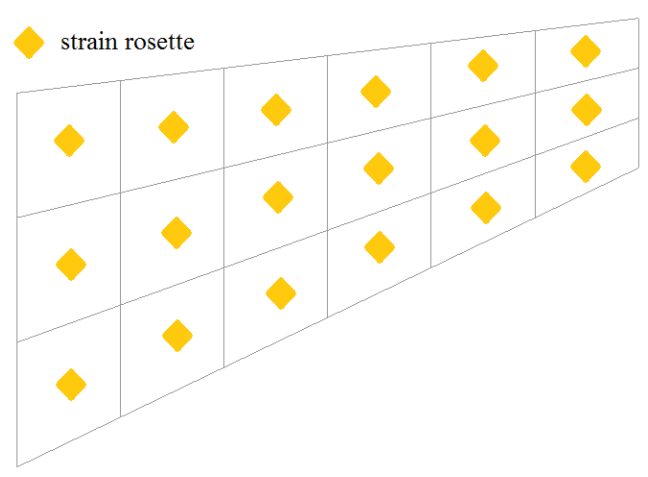

Figure 5. Approximate locations of 18 strain rosettes on quadrilateral plate discretized by $18 \mathrm{iQS} 4$ elements.

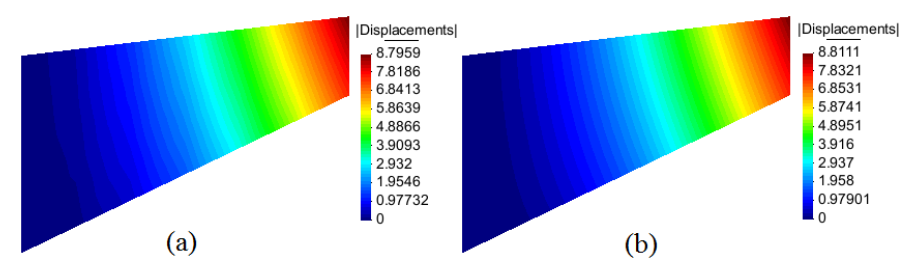

Figure 6. (a) iFEM total displacement distribution of quadrilateral plate (18 iQS4 elements - 18 strain rosettes). (b) Direct FEM total displacement distribution of quadrilateral plate.

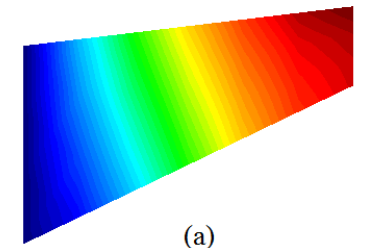

(a)

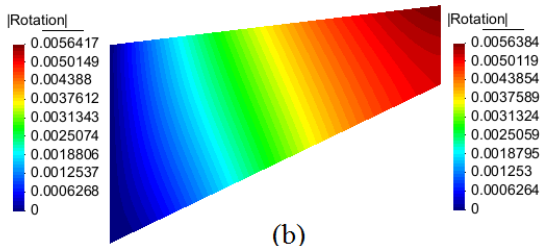

(b)
Figure 7. (a) iFEM total rotation distribution of quadrilateral plate (18 iQS4 elements - 18 strain rosettes). (b) Direct FEM total rotation distribution of quadrilateral plate. 
In the first example, the quadrilateral plate is uniformly discretized by 18 iQS4 elements possessing 168 degrees-of-freedoms (Figure 5). 18 strain rosettes are placed on each element's top surface at centroids as shown in Figure 5. The total displacement and rotation results obtained from iFEM analysis are respectively shown together with the direct FEM results in Figure 6-7. According to the distributions, the error of the maximum displacement and rotation obtained from iFEM solution is less than $0.2 \%$ with respect to the direct FEM results.

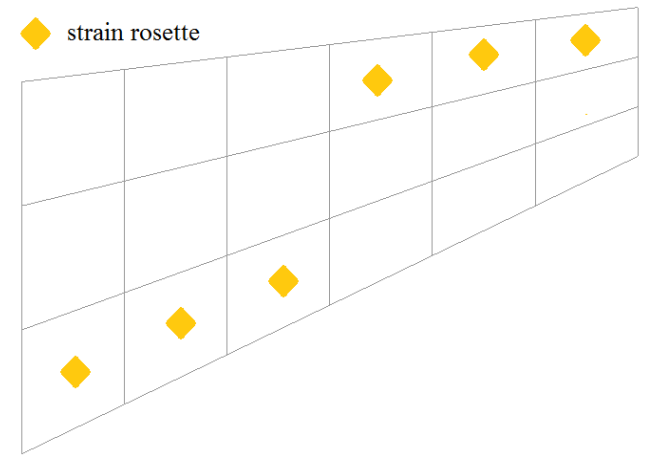

Figure 8. Approximate locations of 6 strain rosettes on quadrilateral plate discretized by 18 iQS4 elements.

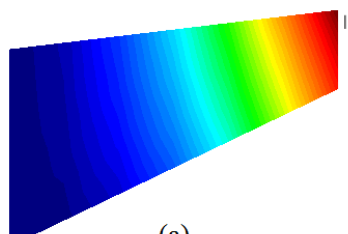

(a)

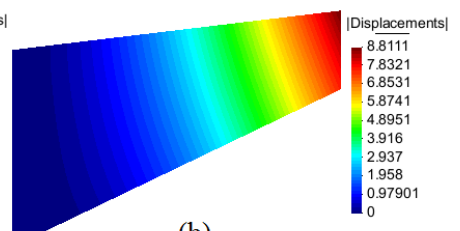

(b) tained from iFEM analysis are respectively compared with direct FEM results as depicted in Figure 9-10. The iFEM-reconstructed displacement and rotation fields almost identically match the reference displacement and rotation fields so that this accuracy confirms the robustness of iFEM framework even if there are missing in-situ strain measurements.

\subsection{Longitudinally and transversely stiffened plate}

Performing SHM of a longitudinally and transversely stiffened plate is crucial since ship structures are generally consisted of various stiffened plates. Inverse quadrilateral shell elements are clearly appropriate to model these types of structures in order to conduct a precise real-time monitoring.

A stiffened square plate that represents the portion of the side of a typical longitudinally and transversely framed tanker is considered to be solved. The plate's edge length and uniform thickness are 3 $\mathrm{m}$ and $15 \mathrm{~mm}$ respectively. Each stiffener has a height of $150 \mathrm{~mm}$ and uniform thickness of $15 \mathrm{~mm}$. The selected material's elastic modulus is $210 \mathrm{GPa}$ with the Poisson's ratio of 0.3. Right, left, upper, and bottom edges of the plate including each stiffener's end edges are clamped. A static uniform transverse pressure of $40 \mathrm{kPa}$ is subjected to the bottom surface of the plate. Applied boundary conditions and isometric view of the stiffened plate are illustrated in Figure 11.

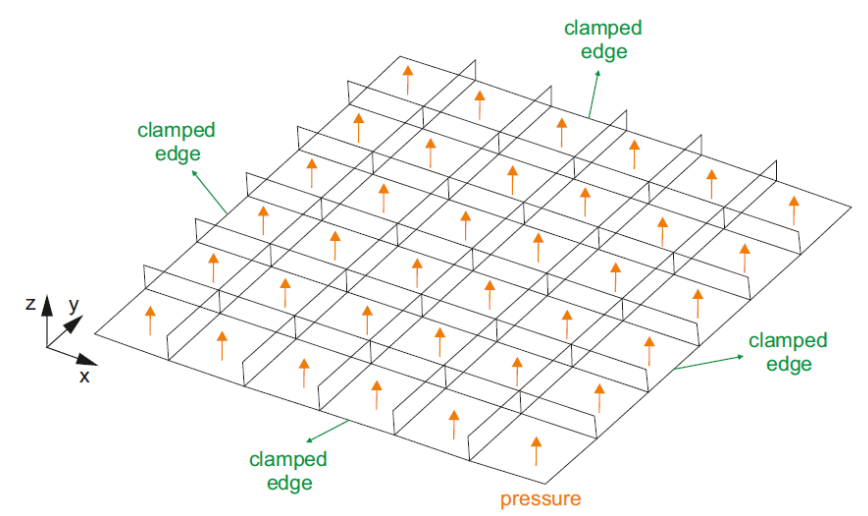

Figure 11. Longitudinally and transversely stiffened plate and its applied boundary conditions.

Initially, a direct FEM convergence study of the stiffened plate using meshes of quadrilateral shell elements is carried out in order to establish an accurate numerical solution. The most refined mesh consisted of 5400 square and uniformly distributed elements, possessing 36966 degrees-of-freedoms. The results of displacement and rotation fields obtained from this convergence study are considered as a reference source to generate the 'experimental' strain measurements (in-situ strain data) used in iFEM analyses.

Two real-time monitoring scenarios of the stiffened plate are respectively executed by using two $10^{-4}$. The total displacement and rotation results ob- 
different iFEM meshes. Although the material properties of the plate and stiffeners are symmetric with respect to the mid-plane, the resulting deformations exhibits both stretching and bending actions due to the complexity of the structure. Hence, the strain rosettes have to be placed on both the top and bottom surfaces of structure for this problem.

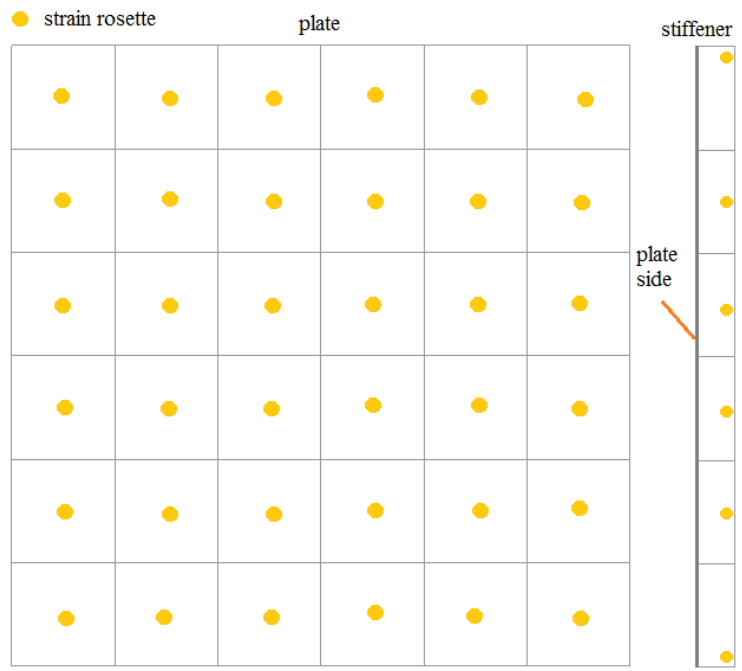

Figure 12. Approximate locations of 96x2 strain rosettes on stiffened plate discretized by 96 iQS4 elements.

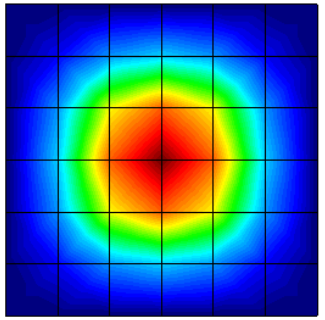

(a)

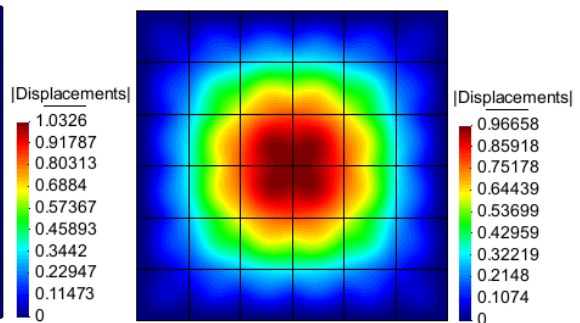

(b)
Figure 13. iFEM total displacement distribution of stiffened plate (96 iQS4 elements - 96x2 strain rosettes). (b) Direct FEM total displacement distribution of stiffened plate.

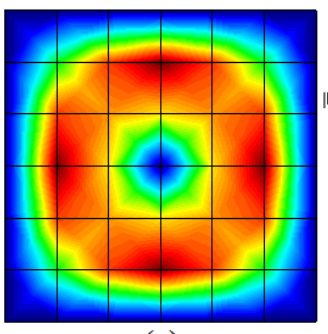

(a)

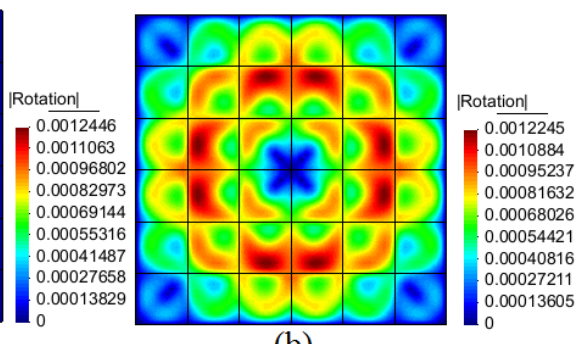

(b)
Figure 14. iFEM total rotation distribution of stiffened plate (96 iQS4 elements - 96x2 strain rosettes). (b) Direct FEM total rotation distribution of stiffened plate.

Firstly, 96 iQS4 elements (36 of them on the plate, 60 of them on the stiffeners) having 1062 degrees-of-freedoms are used to generate the iFEM mesh of the stiffened plate as shown in Figure 12. Strain rosettes are positioned at center of each iQS4 element attached to the plate while each iQS4 ele- ment on each stiffener has strain rosettes placed near to reverse side of the plate as shown in Figure 12. The distribution of reconstructed total displacement and rotation are respectively compared with those obtained from direct FEM analysis as depicted in Figure 13-14. According to the comparisons, the error of the maximum displacement produced by the iFEM solution is approximately $6 \%$ with respect FEM maximum displacement and the results are graphically agreed quite well. Even though the variation of rotation results seems slightly dissimilar for several locations, the maximum rotation found by iFEM analysis is less than $2 \%$ in error compared to the FEM maximum rotation. Moreover, the locations of maximum and minimum rotations on plate found in iFEM and FEM analyses are in good agreement.

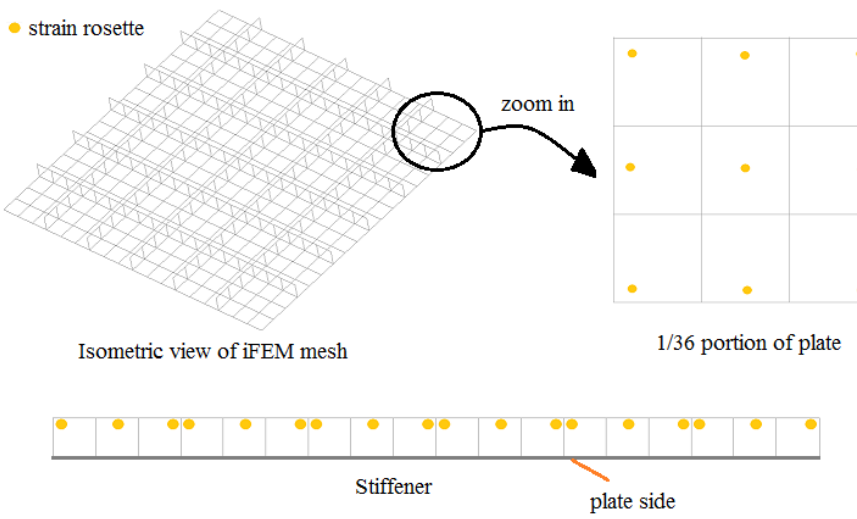

Figure 15. Approximate locations of $504 \times 2$ strain rosettes on stiffened plate discretized by 504 iQS4 elements.

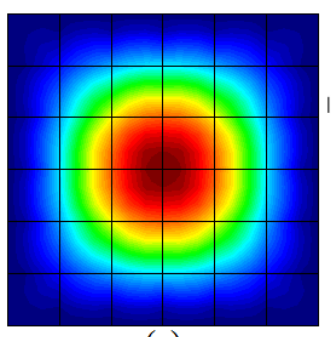

(a)

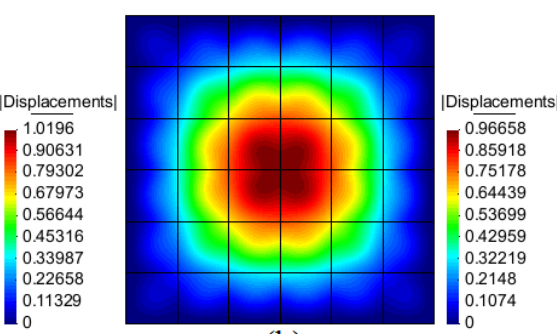

(b)
Figure 16. iFEM total displacement distribution of stiffened plate (504 iQS4 elements - 504x2 strain rosettes). (b) Direct FEM total displacement distribution of stiffened plate.

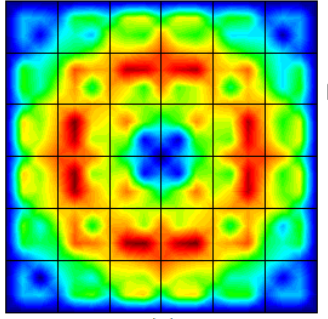

(a)

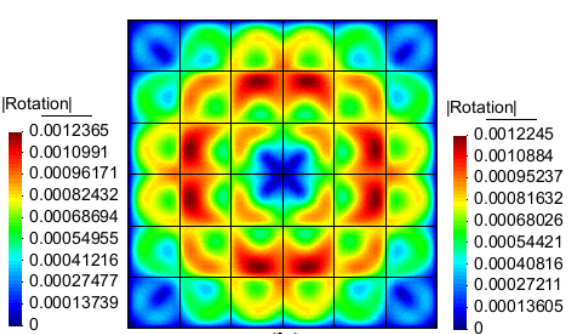

(b)
Figure 17. iFEM total rotation distribution of stiffened plate (504 iQS4 elements - 504x2 strain rosettes). (b) Direct FEM total rotation distribution of stiffened plate. 
In the second iFEM analysis, the plate is uniformly discretized by using 504 iQS4 elements as illustrated in Figure 15 where the approximate locations of the in-situ strain rosettes are included in detail as well. The total number of strain rosettes count as $504 \times 2$, since each iQS4 element is instrumented with a strain rosette on the top and bottom surfaces. The distribution of reconstructed total displacement and rotation presented in Figure 16-17 confirms the high precision of iFEM framework when a finer iFEM mesh including more strain rosettes is used.

\section{CONCLUSIONS}

A revised formulation of the inverse Finite Element Method (iFEM) is presented. The presented iFEM methodology is applicable to perform shape-sensing analyses of plate and shell structures by using the strain data obtained from randomly distributed sensors on the structure. A four-node inverse shell element (iQS4) including hierarchical drilling rotation degree of freedoms is formulated to perform numerical simulations. Application of iFEM to SHM of marine structures is established by using various types of low- and high-fidelity discretization strategies of presented problems. The effect of sensor locations, number of sensors and the discretization of the geometry on solution accuracy are pondered. The numerical results have confirmed that it is still possible to reconstruct sufficiently accurate deformed structural shapes, even if a large amount of strain-sensor data is not available. According to results, it has been agreed that iFEM methodology is promising technology for performing an accurate real-time monitoring of marine structures.

\section{ACKNOWLEDGEMENTS}

The authors would like to acknowledge FP7 project INCASS for financial support.

\section{REFERENCES}

Bathe, K. J. 2006. Finite element procedures. Klaus-Jurgen Bathe.

Cook, R. D. 1994. Four-node 'flat'shell element: drilling degrees of freedom, membrane-bending coupling, warped geometry, and behavior. Computers \& structures, 50(4), 549555.

Cerracchio, P., Gherlone, M., Mattone, M., Di Sciuva, M., \& Tessler, A. 2010. Shape sensing of three-dimensional frame structures using the inverse finite element method. In: Proceedings of 5th European Workshop on Structural Health Monitoring, Sorrento, Italy.

Gherlone, M., Cerracchio, P., Mattone, M., Di Sciuva, M., \& Tessler, A. 2011. Beam shape sensing using inverse finite element method: theory and experimental validation. In:
Proceeding of $8^{\text {th }}$ International Workshop on Structural Health Monitoring, Stanford, CA.

Gherlone, M., Cerracchio, P., Mattone, M., Di Sciuva, M., \& Tessler, A. 2012. Shape sensing of 3D frame structures using an inverse Finite Element Method. International Journal of Solids and Structures, 49(22), 3100-3112.

Gherlone, M., Di Sciuva, M., Cerracchio, P., Mattone, M. C., \& Tessler, A. 2013. The inverse Finite Element Method for shape sensing of aerospace structures. In: Proceedings of $22^{\text {nd }}$ Conference of Italian Association of Aeronautics and Astronautics, Naples, Italy.

Gherlone, M., Cerracchio, P., Mattone, M., Di Sciuva, M., \& Tessler, A. 2014. An inverse finite element method for beam shape sensing: theoretical framework and experimental validation. Smart Materials and Structures, 23(4), 045027.

Liu, P. L., \& Lin, H. T. 1996. Direct identification of nonuniform beams using static strains. International journal of solids and structures, 33(19), 2775-2787.

Maniatty, A., Zabaras, N. J., \& Stelson, K. 1989. Finite element analysis of some inverse elasticity problems. Journal of engineering mechanics, 115(6), 1303-1317.

Maniatty, A. M., \& Zabaras, N. J. 1994. Investigation of regularization parameters and error estimating in inverse elasticity problems. International journal for numerical methods in engineering, 37(6), 1039-1052.

Schnur, D. S., \& Zabaras, N. J. 1990. Finite element solution of two-dimensional inverse elastic problems using spatial smoothing. International Journal for Numerical Methods in Engineering, 30(1), 57-75.

Tessler, A., \& Hughes, T. J. 1983. An improved treatment of transverse shear in the Mindlin-type four-node quadrilateral element. Computer methods in applied mechanics and engineering, 39(3), 311-335.

Tessler, A., Riggs, H. R., Freese, C. E., \& Cook, G. M. 1998. An improved variational method for finite element stress recovery and a posteriori error estimation. Computer methods in applied mechanics and engineering, 155(1), 15-30.

Tessler, A., Riggs, H. R., \& Dambach, M. 1999. A novel fournode quadrilateral smoothing element for stress enhancement and error estimation. International journal for numerical methods in engineering, 44(10), 1527-1541.

Tessler, A., \& Spangler, J.L. 2003. A variational principal for reconstruction of elastic deformation of shear deformable plates and shells, NASA TM-2003-212445.

Tessler, A., \& Spangler, J.L. 2004. Inverse FEM for full-field reconstruction of elastic deformations in shear deformable plates and shells. In: Proceedings of $2^{\text {nd }}$ European Workshop on Structural Health Monitoring, Munich, Germany.

Tessler, A., \& Spangler, J. L. 2005. A least-squares variational method for full-field reconstruction of elastic deformations in shear-deformable plates and shells. Computer methods in applied mechanics and engineering, 194(2), 327-339.

Tessler, A., Spangler, J. L., Gherlone, M., Mattone, M., \& Di Sciuva, M. 2011. Real-Time characterization of aerospace structures using onboard strain measurement technologies and inverse finite element method. In: Proceedings of the $8^{\text {th }}$ International Workshop on Structural Health Monitoring, Stanford, CA.

Tessler, A., Spangler, J. L., Gherlone M., Mattone M., \& Di Sciuva, M. 2012. Deformed shape and stress reconstruction in plate and shell structures undergoing large displacements: application of inverse finite element method using fiberbragg-grating strains. In: Proceedings of $10^{\text {th }}$ World Congress on Computational Mechanics, Sao Paulo, Brazil.

Tikhonov, A. N., \& Arsenin, V. Y. 1977. Solutions of ill-posed problems. Winston, Washington, DC.

Quach, C.C., Vazquez, S.L., Tessler, A., Moore, J.P., Cooper, E.G., \& Spangler, J.L. 2005. Structural anomaly detection 
using fiber optic sensors and inverse finite element method. In: Proceedings of AIAA Guidance, Navigation, and Control Conference and Exhibit, San Francisco, California.

Vazquez, S.L., Tessler, A., Quach, C.C., Cooper, E.G., Parks, J., \& Spangler J.L. 2005. Structural health monitoring using high-density fiber optic strain sensor and inverse finite element methods, NASA TM-2005-213761. 